\title{
Report of an international collaborative study to establish the suitability of using modified ATP assay for viable count of BCG vaccine
}

\author{
Mei M. Ho ${ }^{\text {a,*}}$, Kevin Markey ${ }^{a}$, Peter Rigsby ${ }^{b}$, Sten E. Jensen ${ }^{1}$, Sunil Gairola ${ }^{1}$, Masaaki Seki ${ }^{1}$, \\ Luiz R. Castello-Branco ${ }^{1}$, Yolanda López-Vidal ${ }^{1}$, Ivana Knezevic ${ }^{c}$, Michael J. Corbel ${ }^{\text {a }}$ \\ a Bacteriology Division, NIBSC, South Mimms, Potters Bar, Hertfordshire EN6 3QG, UK \\ b BioStatistics, NIBSC, South Mimms, Potters Bar, Hertfordshire EN6 3QG, UK

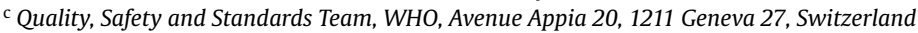

\section{A R T I C L E I N F O}

\section{Article history:}

Received 5 June 2008

Accepted 10 June 2008

Available online 27 June 2008

\section{Keywords:}

BCG

ATP

Viability

\begin{abstract}
A B S T R A C T
As part of the World Health Organisation (WHO) initiative to update the current requirements for BCG vaccine a collaborative study was carried out to establish the robustness, reproducibility and the suitability of the modified ATP assay. This assay was developed by Statens Serum Institut, Denmark, as a potential replacement of the method for detection of viable counts of BCG vaccine which is routinely used as a quality control test for lot release. Two BCG preparations, of same strain but different production methods, were tested. For each preparation, two different storage conditions of -20 or $37^{\circ} \mathrm{C}$ were used in order to establish the suitability of this assay for testing heat-treated BCG vaccine as in the temperature stability test. The lyophilised BCG samples were tested using the ATP reagents from the same source and same principle of testing but some procedural modifications were allowed to accommodate different equipment and resource availability in different laboratories. Data from four laboratories showed that the heat-treated BCG samples contained significantly lower ATP content per sample than the untreated control stored at $-20^{\circ} \mathrm{C}$. Three laboratories gave consistent mean ATP contents, especially for control samples, even with variations in testing protocol. The present study showed that this modified ATP assay is very robust and can be reproducible. Once the correlation of cultural viable count and ATP content of a BCG vaccine product has been established, this rapid alternative assay may be used to monitor BCG viable count. Due to the fact that this study was small, further investigation is planned. A collaborative study will be carried out using this modified ATP assay in parallel with the cultural viable count method in the establishment of the replacement of the WHO International Reference Preparation of BCG vaccine.
\end{abstract}

(ㄷ) 2008 Elsevier Ltd. All rights reserved.

\section{Introduction}

BCG vaccine is based on a live attenuated strain of Mycobacterium bovis. The viability of the organisms is essential for the stimulation of a protective immune response and monitoring viable count is an integral part of quality control. Although measurement of viable count is not in itself an assay of potency, it has been used as a surrogate of BCG potency [1,2]. The cultural viable count assay, often known as Colony Forming Unit (CFU) test, is problematic and very time consuming, as mycobacteria are very slow growing organisms, thus re-testing of bulk samples before formulation is usually impossible. The slowness, poor reproducibility and high variability of test results are the main driving forces for manufacturers and control laboratories to look for a rapid, more

\footnotetext{
* Corresponding author. Tel.: +44 1707 641434; fax: +44 1707641054.

E-mail address: mho@nibsc.ac.uk (M.M. Ho).

1 Other participants in this study as listed in Appendix A.
}

reproducible alternative viable count assay. This issue has been addressed in recent WHO consultation meetings on characterisation and improvement of the quality control of BCG vaccines $[3,4]$. An improved ATP luminescence assay has been discussed and promoted as a choice of alternative viable count assay for collaborative study.

This modified ATP assay, including the step of overnight incubation of reconstituted BCG in suitable culture medium, has been introduced and developed by Statens Serum Institut (SSI) as an alternative rapid assay for viable count of BCG vaccine [5]. The assay was tested at NIBSC as part of its evaluation. The method for estimation of ATP content in BCG vaccine is based on the reaction of firefly luciferase with ATP which results in bioluminescence production [6]. The intensity of light emission measured is directly proportional to ATP content in the sample which can be estimated by using the ATP standards for calibration.

As part of the WHO initiative to update the current requirements for BCG vaccine [1] and to establish a replacement for the WHO First International Reference Preparation of BCG vaccine, a collaborative 
study was carried out to evaluate the robustness, reproducibility and the suitability of the modified ATP assay for viable counts of BCG vaccine. Many alternative biochemical assays for BCG viable counts failed to show significant differences in viability in temperature stability tests. In this study, it is important to show that this modified ATP assay can detect significant differences in viability of lyophilised BCG samples stored under control $\left(-20^{\circ} \mathrm{C}\right)$ or heat-treated (at $37^{\circ} \mathrm{C}$ for 4 weeks) conditions.

\section{Materials and methods}

\subsection{Study materials}

Two BCG vaccine preparations of the same strain (Danish 1331) but different production methods were provided by Satens Serum Institut, Denmark as test samples. Lyophilised BCG in ampoule (containing $2 \mathrm{mg}$ lyophilised BCG) and vial (containing $0.75 \mathrm{mg}$ lyophilised BCG) preparations were coded (ampoule A1-A20; vials V1-V20) and blinded at NIBSC. The pre-incubation at $37^{\circ} \mathrm{C}$ for 4 weeks of ten randomly selected ampoules and vials from each sample group (Table 1) was performed at NIBSC. After heat treatment, samples were stored at $-20^{\circ} \mathrm{C}$. All test samples were shipped on dry ice and recipients were advised to store them at $-20^{\circ} \mathrm{C}$ until testing.

\subsection{Participants}

Seven laboratories including four manufacturers, one national control laboratory and two research laboratories agreed to participate in the study. However, one research laboratory withdrew its participation without testing the samples due to the failure of essential equipment. The six participants were assigned with a code number not corresponding to the order of listing and are detailed in Appendix A.

\subsection{Study design and testing protocol}

Participants were requested to test the two sets of samples without any information on which ampoules/vials had been heattreated, using the modified ATP assay with protocol provided. Some minor modifications of the test method were allowed to accommodate different equipment (e.g. luminescence plate or cuvette reader) availability. Results were recorded and data sent to NIBSC for collation and statistical analysis.

The modified ATP assay was developed by SSI [5] and the same method was recommended, except that for the statistical purposes of this WHO collaborative study, additional dilutions were included in the testing protocol with the intention of making statistical analysis more accurate. It was recommended to prepare three different dilutions from each vial/ ampoule and with triplicates for each reading. In brief, lyophilised BCG samples were reconstituted with $1 \mathrm{ml}$ Dubos medium (SSI Diagnostica, Denmark) and the BCG suspensions were incubated at $37^{\circ} \mathrm{C}$ for $22-26 \mathrm{~h}$. Dilutions of overnight BCG culture were prepared in pre-warmed Dubos medium as undiluted, $1: 2$ and 1:5 dilutions. ATP extraction was performed by adding $100 \mu \mathrm{l}$ of BCG suspension directly to the pre-heated $500 \mu \mathrm{l}$ Tris-EDTA (TE) buffer at $96-98^{\circ} \mathrm{C}$ and incubating at this temperature for exactly $6 \mathrm{~min}$. Negative control was prepared as samples using Dubos medium without BCG. After heat treatment, the resulting extracts were required to cool to room temperature before addition of ATP reagent (BioThema AB, Sweden). ATP standard (BioThema $A B$, Sweden) in serial dilutions was prepared and $100 \mu \mathrm{l}$ of each diluted standard was added to $500 \mu$ l of TE buffer without heating. The ATP extract or standard was then added with appropriate amount of ATP reagent and the bioluminescence of the samples and ATP standards was measured in a luminescence counter at about 5 min delay after addition of ATP reagent.

\subsection{Calculation and statistical methods}

Standard curves were generated by linear regression of $\log _{10}$ response on $\log _{10}$ concentration of ATP standard. Responses for the test samples were converted to results in pmol (of ATP)/ $100 \mu \mathrm{l}$ (of reconstituted BCG sample in $1 \mathrm{ml}$ ) using the fitted regression lines of ATP standard. All results have been combined as unweighted geometric means with variability expressed using geometric coefficients of variation (GCV). All comparisons have been made by unpaired $t$-test or analysis of variance using logtransformed results.

\section{Results}

Six data sets were collected from participants. Among them, two sets of data were not used and considered invalid for direct comparison. Laboratory 5 performed the assay by reconstituting the BCG samples in $2 \mathrm{ml}$ of Sauton medium and did not perform the crucial step of overnight incubation of the reconstituted BCG samples at $37^{\circ} \mathrm{C}$ before the assay of ATP content. Laboratory 6 performed the ATP extraction step at $94^{\circ} \mathrm{C}\left(96^{\circ} \mathrm{C}\right.$ was set as the lower limit) and the data showed that all luminescence readings (except one ampoule) of tested samples fell well below the lowest point $(<0.31 \mathrm{pmol} / 100 \mu \mathrm{l})$ of the ATP standard calibration curves. Thus the ATP content of the samples could not be estimated correctly. Therefore, these results were excluded and only four data sets were used in direct comparisons. With the data used for direct comparison, only two participants performed the ATP assay with three different dilutions and three sample readings each as listed in the protocol provided.

Table 1

The coding system of BCG samples randomly selected for pre-incubation at $37^{\circ} \mathrm{C}$ for four weeks

\begin{tabular}{|c|c|c|c|c|c|c|c|c|c|c|}
\hline \multicolumn{11}{|l|}{ Ampoule samples } \\
\hline No. & $\mathrm{A} 1$ & A2 & A3 & A4 & A5 & A6 & A7 & A8 & A9 & A10 \\
\hline $37^{\circ} \mathrm{C}, 4$ weeks & - & + & + & - & - & - & + & - & + & + \\
\hline No. & A11 & A12 & A13 & A14 & A15 & A16 & A17 & A18 & A19 & A20 \\
\hline $37^{\circ} \mathrm{C} 4$ weeks & + & - & - & + & - & + & + & - & - & + \\
\hline \multicolumn{11}{|l|}{ Vial samples } \\
\hline No. & V1 & V2 & V3 & V4 & V5 & V6 & V7 & V8 & V9 & V10 \\
\hline $37^{\circ} \mathrm{C}, 4$ weeks & - & + & - & + & - & - & + & + & + & - \\
\hline No. & V11 & V12 & V13 & V14 & V15 & V16 & V17 & V18 & V19 & V20 \\
\hline $37^{\circ} \mathrm{C} 4$ weeks & - & - & - & + & + & + & + & - & + & - \\
\hline
\end{tabular}

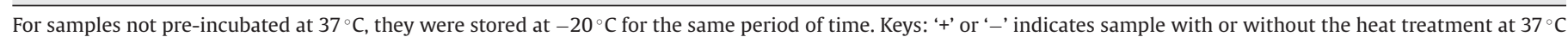
for four weeks, respectively. 
Table 2

Details of the modified ATP assay conditions used by participating laboratories in the collaborative study

\begin{tabular}{|c|c|c|c|c|}
\hline Laboratory & 1 & 2 & 3 & 4 \\
\hline Culture medium used & Middlebrook 7H9 & Dubos & Dubos & Dubos \\
\hline No. of samples test per experiment & $6-10$ & 10 & $5-6$ & $2-4$ \\
\hline Samples prepared per ampoule/vial & 1 (undiluted) & 1 (undiluted) & 3 (undiluted, $1: 2,1: 5$ ) & 3 (undiluted, $1: 2,1: 5$ ) \\
\hline Luminescence reading per sample & 1 & 1 & 3 & 3 \\
\hline Luminescence reader & Cuvette & Cuvette & Plate & Cuvette \\
\hline
\end{tabular}

Table 3

Summary of results of ATP content in undiluted BCG samples

\begin{tabular}{|c|c|c|c|c|}
\hline Laboratory & Set & Estimate mean content of ATP $(\mathrm{pmol} / 100 \mu \mathrm{l})$ & 95\% C.I. & GCV (\%) \\
\hline \multirow[t]{4}{*}{1} & Vials & 2.85 & $2.70-3.06$ & 9 \\
\hline & Vials $\left(37^{\circ} \mathrm{C}\right)$ & 1.31 & $1.14-1.50$ & 18 \\
\hline & Ampoules & 6.88 & $6.42-7.32$ & 9 \\
\hline & Ampoules $\left(37^{\circ} \mathrm{C}\right)$ & 4.46 & $3.90-5.10$ & 21 \\
\hline \multirow[t]{4}{*}{2} & Vials & 4.69 & $4.37-5.02$ & 10 \\
\hline & Vials $\left(37^{\circ} \mathrm{C}\right)$ & 1.80 & $1.70-1.90$ & 8 \\
\hline & Ampoules & 15.92 & $11.56-21.93$ & 57 \\
\hline & Ampoules $\left(37^{\circ} \mathrm{C}\right)$ & 8.29 & $5.14-13.35$ & 95 \\
\hline \multirow[t]{4}{*}{3} & Vials & 2.85 & $2.21-3.69$ & 43 \\
\hline & Vials $\left(37^{\circ} \mathrm{C}\right)$ & 0.95 & $0.74-1.23$ & 41 \\
\hline & Ampoules & 6.82 & $5.77-8.05$ & 26 \\
\hline & Ampoules $\left(37^{\circ} \mathrm{C}\right)$ & 3.47 & $2.69-4.49$ & 43 \\
\hline \multirow[t]{4}{*}{4} & Vials & 2.92 & $2.39-3.57$ & 32 \\
\hline & Vials $\left(37^{\circ} \mathrm{C}\right)$ & 1.02 & $0.87-1.20$ & 24 \\
\hline & Ampoules & 5.73 & $4.52-7.26$ & 39 \\
\hline & Ampoules $\left(37^{\circ} \mathrm{C}\right)$ & 2.70 & $1.97-3.69$ & 50 \\
\hline
\end{tabular}

Keys: C.I. = confidence interval; GCV = geometric coefficients of variation.

The testing protocol was modified by individual laboratories as detailed in Table 2. For example, Middlebrook 7H9 culture medium was used instead of Dubos medium as recommended. Between two and ten ampoules or vials were tested with a single run of a standard curve. All laboratories used a luminescence cuvette reader except one which used a plate reader.

One assay by Laboratory 4 did not include a standard curve, so no result is shown for one vial and one ampoule. Although several of the ampoules tested by Laboratory 2 were above the range of the standard curve, it was considered as a relevant set of data and all results have been included in this report.

The results calculated using only undiluted test samples are summarised in Table 3 and shown in Fig. 1. Among the four sets of data used for comparison, some differences in the mean ATP contents were noted among laboratories (Table 3). For the untreated/control samples Participants No. 1, 3 and 4 obtained consistent results with no significant differences detected between the laboratories. For the heat-treated samples, only Participant No. 3 and 4 obtained consistent results and showed no significant differences between the two laboratories. Results from Participant No. 1-4 all showed that heat-treated samples contained significantly lower ATP content per $100 \mu$ l of test sample than the untreated control stored at $-20^{\circ} \mathrm{C}$. This was highly significant $(p<0.001)$ in all cases except for the ampoule sample of Laboratory $2(p<0.05)$, where one heat-treated ampoule gave an unusually high result. The difference in results between heat-treated and untreated was greater for vials (55-67\% less) than ampoules (35-53\% less).

Laboratory 3 and 4 also tested the diluted test samples 1:2 and 1:5. For comparison, a summary of these results is given in Table 4 . The data showed that significantly higher ATP content was estimated after vials were diluted 1:2 (around 15\% higher) or 1:5 (around 25\% higher). Higher ATP content was also obtained for the ampoules, but this was only found to be significant at Laboratory 4 and not at Laboratory 3. There is no clear explanation for this

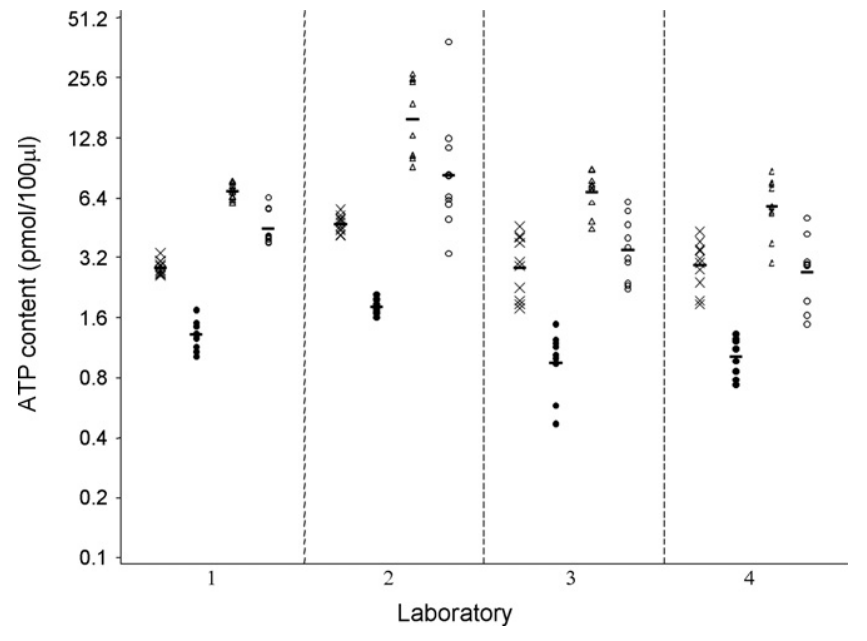

Fig. 1. Summary of results of estimate ATP content in undiluted BCG samples. Ten samples per group were tested in the modified ATP assay. Individual results were plotted with geometric mean. Keys: ' $\mathrm{X}$ ' vial sample stored at $-20^{\circ} \mathrm{C}$; ' $\bullet$ ' vial sample pre-incubated at $37^{\circ} \mathrm{C}$ for 4 weeks; ' $\Delta$ ' ampoule sample stored at $-20^{\circ} \mathrm{C}$; ' $\bigcirc$ ' ampoule sample pre-incubated at $37^{\circ} \mathrm{C}$ for 4 weeks; '-' geometric mean.

unexpected dilution effect on higher ATP content estimation from diluted samples. This dilution effect was also observed in the raw data (not shown) from Laboratory 5 though its data were not valid for direct comparison in this study.

\section{Discussion}

This modified ATP assay is simple and easy to perform. Among all four sets of data used for direction comparison, it is clear that this assay is capable of detecting the significant reduction of viability in lyophilised BCG vaccine (in terms of ATP content) stored 
Table 4

Summary of results of ATP content obtained using undiluted and diluted BCG samples

\begin{tabular}{|c|c|c|c|c|}
\hline \multirow[t]{2}{*}{ Laboratory } & \multirow[t]{2}{*}{ Set } & \multicolumn{3}{|c|}{ Estimate mean content of ATP in pmol/ $100 \mu \mathrm{l}$ (95\% C.I.) } \\
\hline & & Undiluted & $1: 2$ & $1: 5$ \\
\hline 3 & $\begin{array}{l}\text { Vials } \\
\text { Vials }\left(37^{\circ} \mathrm{C}\right) \\
\text { Ampoules } \\
\text { Ampoules }\left(37^{\circ} \mathrm{C}\right)\end{array}$ & $\begin{array}{l}2.85(2.21-3.69) \\
0.95(0.74-1.23) \\
6.82(5.77-8.05) \\
3.47(2.69-4.49)\end{array}$ & $\begin{array}{l}3.56^{*}(2.72-4.65) \\
1.09^{* *}(0.83-1.43) \\
7.06(5.85-8.50) \\
3.33(2.62-4.22)\end{array}$ & $\begin{array}{l}3.67^{* *}(2.73-4.94) \\
1.10^{* *}(0.85-1.43) \\
7.30(5.77-9.23) \\
3.60(2.76-4.70)\end{array}$ \\
\hline 4 & $\begin{array}{l}\text { Vials } \\
\text { Vials }\left(37^{\circ} \mathrm{C}\right) \\
\text { Ampoules } \\
\text { Ampoules }\left(37^{\circ} \mathrm{C}\right)\end{array}$ & $\begin{array}{l}2.92(2.39-3.57) \\
1.02(0.87-1.20) \\
5.73(4.52-7.26) \\
2.70(1.97-3.69)\end{array}$ & $\begin{array}{l}3.26^{*}(2.70-3.94) \\
1.25^{* *}(1.03-1.50) \\
5.97(4.32-8.25) \\
3.02^{*}(2.28-3.99)\end{array}$ & $\begin{array}{l}3.94^{* *}(3.20-4.86) \\
1.31^{* *}(1.08-1.60) \\
6.73^{*}(5.09-8.89) \\
3.30^{* *}(2.65-4.10)\end{array}$ \\
\hline
\end{tabular}

Keys: C.I. = confidence interval.

Significantly larger than undiluted $(p<0.05)$.

*** Significantly larger than undiluted $(p<0.01)$.

at $37^{\circ} \mathrm{C}$ for 4 weeks as in the temperature stability test when compared with control samples (Fig. 1). The assay is robust as results from three laboratories out of four valid data sets were consistent, especially for control samples, even with variations in testing protocol such as different culture media and luminescence equipment were used. This modified ATP assay is by far more reproducible than the cultural method, as the GCV within each sample group is relatively small (up to about $50 \%$ except one; Table 3 ) when compared with the cultural methods in which the GCV can generally go up to $100-200 \%$ (data not included).

However, there is a need to establish the correlation of cultural viable counts with ATP content of each BCG product before this rapid assay can be applied for monitoring BCG viable counts. This correlation was not assessed in this study. It is important for each individual laboratory to establish this correlation by experimental determinations for each BCG product. The ATP standard should be kept at $-20^{\circ} \mathrm{C}$ in a sealed vial for long-term storage (usually for 2 years as recommended by manufacturer) to avoid changes in concentration due to evaporation of water content. A replacement batch of ATP standard should be tested against the current ATP standard before the expiry date to ensure the consistency of the calibration.

A plan is underway to further advance this collaborative study of modified ATP assay for BCG viable count. It will involve BCG vaccine of different substrains and production methods, and this rapid assay will be carried out in parallel with the cultural viable count method. These future studies will form part of the project for the establishment of the replacement of the First International Reference Preparation of BCG vaccine which is more than 40 years old. The outcome of these future studies will be reported to the Expert Committee on Biological Standardization (ECBS) for further consideration and address the suitability of this rapid assay for recommendation to monitor BCG viable counts for manufacturers and national control laboratories.

\section{Acknowledgements}

Special thanks are to Statens Serum Institut, Denmark for the supply of BCG preparations, methodology and scientific inputs in this study. The participating laboratories and scientists who contributed their results and to the discussion of this study are also acknowledged. This collaborative study was supported by WHO. The part of the collaboration in Universidad Nacional Autónoma de México was funded by Project SDEI.PTID. 05.04 .

\section{Appendix A. List of participants}

Dr. Luiz R.R. Castello-Branco, Fundação Ataulpho de Paiva, Av. Pedro II, 260, São Cristovão, Rio de Janeiro, Brazil.

Dr. Sunil Gairola, Serum Institute of India Ltd., 212/2, Hadaspar, Pune-411028, India.

Dr. Mei M. Ho and Dr. Kevin Markey, Bacteriology Division, NIBSC, South Mimms, Potters Bar, Herts. EN6 3QG, UK.

Dr. Sten E. Jensen, QC Department, B. 47/312, Statens Serum Institut, Artillerivej 5, DK-2300, Copenhagen, Denmark.

Dr. Masaaki Seki, Japan BCG Laboratory, 3-1-5, Matsuyama, Kiyose City, Tokyo 204-0022, Japan.

Dr. Yolanda López-Vidal, Departamento de Microbiología y Parasitología, Facultad de Medicina, Universidad Nacional Autónoma de México.

\section{References}

[1] Requirements for dried BCG vaccine. WHO Expert committee on Biological Standardization. Annex 2. WHO Technical Report Series No. 745; 1987. p. 60-92.

[2] BCG vaccine, Freeze-dried. European Pharmacopoeia. Directorate for the Quality of Medicines of the Council of Europe (EDQM). 6th ed. Strasbourg, Cedex, France; 2008 [01/2008: 0163].

[3] Ho MM, Corbel MJ, Knezevic I, Roumiantzeff M.Report on a WHO consultation on the characterisation of BCG vaccines. Vaccine 2005;23: 5700-4.

[4] Knezevic I, Corbel MJ.WHO discussion on the improvement of the quality control of BCG vaccines. Vaccine 2006;24:3874-7.

[5] Jensen E, Hubrechts P, Klein B, Haslov KR. Development and validation of an ATP method for rapid estimation of viable units in lyophilized BCG Danish 1331 vaccine. Biologicals 2008; in press.

[6] Lundin A. Use of firefly luciferase in ATP-related assays of biomass, enzymes, and metabolites. Methods Enzymol 2000;305:346-70. 